\title{
INTERPRETING THE MAIN POWER CHARACTERISTICS CHOICE OF THE WHEEL VEHICLES GUIDED CUSHIONING SYSTEM
}

\author{
Bogdan Sokil $^{1}$, Oleg Lyashuk², Mariya Sokil ${ }^{3}$, Yuriy Vovk ${ }^{4, *}$, Volodymyr Dzyura ${ }^{4}$, Victor Aulin ${ }^{5}$, Roman Khoroshun² \\ ${ }^{1}$ Department of Engineering Mechanics, Hetman Petro Sahaidachnyi National Army Academy, Lviv, Ukraine \\ ${ }^{2}$ Department of Automobiles, Ternopil Ivan Puluj National Technical University, Ternopil, Ukraine \\ ${ }^{3}$ Department of Transport Technologies, Lviv Polytechnic National University, Lviv, Ukraine \\ ${ }^{4}$ Department of Transport Technologies and Mechanics, Ternopil Ivan Puluj National Technical University, Ternopil, \\ Ukraine \\ ${ }^{5}$ Department of Maintenance and Repair of Machines, Central Ukrainian National Technical University, Kropyvnytskyi, \\ Ukraine \\ *E-mail of corresponding author: vovkyuriy@ukr.net
}

\begin{abstract}
Resume
The method of investigation of the non-linear vibrations of the wheel vehicles with the guided cushioning system has been developed. It is based on the idea of the perturbation methods combined with the theory of special periodic Atebfunctions. All these made possible to obtain analytical relations, which describe the characteristic features of the cushioning area vibrations. These relations can be the basis for creation of the software product of the guided cushioning system with the purpose to minimize the dynamic loadings on the transported people and cargo and to increase the stability characteristics of the vehicle movement along the curvilinear areas of the bump road.
\end{abstract}

Available online: https://doi.org/10.26552/com.C.2021.2.B139-B149

\section{Article info}

Received 31 August 2020

Accepted 25 September 2020

Online 23 February 2021

\section{Keywords:}

non-linear vibration,

Ateb-function,

stability,

vehicle,

guided cushioning system

\section{Introduction}

Guided or semi-guided suspension system [1-4] has been widely used for improving of the wheel vehicle (WV) smooth movement for the last decades. It makes possible to adjust the main characteristics of the suspension system so that the dynamic loading on people or cargo would be minimum. The basis of "adjustment" of these characteristics is the physical and corresponding to it mathematical model of the WV dynamics along the bump road, as well as the response of the latter to this or that type of motion perturbation. However, the mathematical instrument for the analytical investigation of the WV dynamics under their different physical models [5-6] is developed generally for the small vibrations under linear dependence or recovering forces on the deformation of the elastic elements (tires in particular) or the damper devices deformation rate. The adaptive control process is carried out in addition to the speed of the spring part, which is an important special case of the regulated suspension [6-9]. In addition, it is necessary to investigate the relationship between the parameters $v_{1}$ and $v_{2}$ for which the nonlinear suspension has similar characteristics to the linear one. In some papers, the method of the analytical investigations of the non-linear elastic forces of absorbers on vertical rolling vibrations and stability of the vehicle's movement along the curvilinear parts of the road has been developed only for the simplest physical and corresponding non-linear mathematical models of the WV dynamics [7, 10-12]. The static deformation and nonlinearity of the system are the main parameters for progressive (regressive) suspensions. That suspension is more comfortable for transportation of dangerous goods and passengers.

The obtained results, which can be the basis for creation of the software of the guided suspension, show that the elastic absorbers, with non-linear law of the recovering force change, affect not only the numerical characteristics of the cushioning mass (CM) vibrations, but provide them with the qualitative new property - their own frequency depends on the amplitude, as well. Thus, the WV with such a cushioning system along the road with the ordered bumps obtains new characteristics. If one is to look at the process from a mathematical point of view, it is non-periodic, since the oscillations amplitude of the damping device due to the impact of the obstacle attenuates and the natural frequency of oscillations depends on its amplitude. These cyclic periodic signals have been outlined by [13]. Furthermore, the critical 


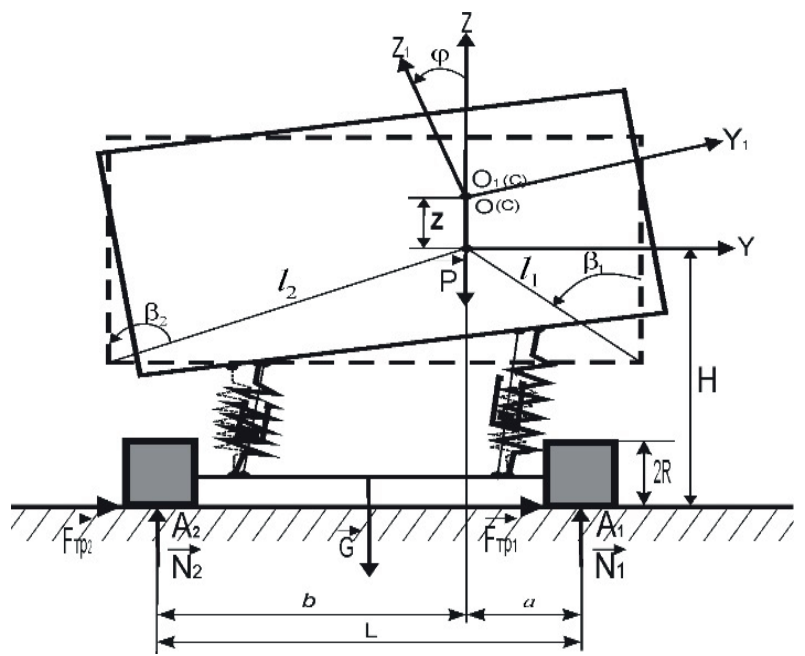

Figure 1 Physical model for investigation of the WV vibrations with the non-conservative characteristics of the cushioning system

speed of the stable motion along the curvilinear areas of the road sufficiently decreases, which is caused by the CM vibrations.

The objective of the work was to obtain the analytical dependencies, which could be the basis for creation of the software product for the more general law than that, for example in [7], of the non-linear recovering force of elastic absorbers, non-conservative in particular.

\section{Materials and methods}

For the WV, physical model of which is presented in Figure 1 and consists of the cushionless - 1, cushioned - 2 parts interrelating between each other by the elastic absorbers - 3 and damping devices - 4 , it is necessary to obtain such analytical dependencies, which would be the basis for creation of the software product of the guided cushioning system with the purpose:

- to minimize the dynamic loadings on transported people and cargo;

- to provide maximum speed of stable motion during the WV manoeuvring, going round the bump or along the curvilinear areas of the rod.

The main assumptions, as to the physical model and the motion of the investigated object, are:

- all the points of the cushioned mass move in the vertical surfaces perpendicular to the speed vector of the WV movement, the latter being constant;

- the mass of the cushionless area is sufficiently smaller than that cushioned one and is being ignored while solving a task;

- force characteristics of the right-hand and left-hand sides of the cushioning system are similar and are described by dependencies $F_{i}\left(\Delta_{i}, \frac{d \Delta_{i}}{d t}\right)=\left(\alpha_{i}+\chi_{i}\left(\frac{d \Delta_{i}}{d t}\right)^{v_{1}}\right) \Delta_{i}^{v_{2}+1} \quad$ - the absorbers elastic force; $R_{i}\left(\frac{d \Delta_{i}}{d t}\right)=\gamma_{i}\left|\frac{d \Delta_{i}}{d t}\right|^{s} \frac{d \vec{\Delta}_{i}}{d t}$ - the damper devices resistance force, in which $\alpha_{i}$, $\beta_{i}, \gamma_{i} v_{j}$ - constants, their values being such as to provide the CM oscillatory motion (they will be analyzed below), $\Delta$ and $d \vec{\Delta}_{i} / d t$ - deformation and the rate of deformation of the elastic absorbers or damping devices;

- maximum values of the damping devices resistance forces are sufficiently smaller than those maximum values of the absorbers elastic forces;

- maximum deformations of the elastic forces are much smaller than those of maximum elastic deformations, because of that they are ignored;

- The CM perturbation is taken into account by the initial conditions in certain mathematical models;

- horizontal displacements of the WV CM and WV mass centre are much smaller than the vertical ones and can be ignored.

Presented above makes possible to state that the relative WV CM motion is plane-parallel, so its position is specified by the position of the mass centre (point 0 ) and the angle of rotation $\varphi(t)$ of the mentioned part around the centre $O$. Position of the mass centre at any time (taking into account the mentioned above restrictions) can be easily found, relatively the static position by the coordinate $-z(t)$. Thus, to build the mathematical model of the CM relative motion the 2-nd order Lagrange equation can be used. According to the chosen generalized coordinates the CM kinetic energy in its relative motion $\mathrm{T}$ looks like

$T=M \frac{\dot{z}^{2}(t)}{2}+I_{C} \frac{\dot{\varphi}^{2}(t)}{2}$,

where $M$-mass of the cushioned area, $I_{C}$ - its inertia moment with respect to the lateral axis, which crosses the masses centre and is parallel to the transient motion vector speed.

As to the generalized forces, which correspond to the generalized chosen coordinates, they can be found, if they are primarily expressed by the WV geometric parameters and mentioned coordinates:

- vectors of relative displacements $\left(\vec{\Delta}_{1}, \vec{\Delta}_{2}\right)$ of the elastic absorbers connection points to WV from its initial position to the arbitrary one 


$$
\begin{aligned}
& \vec{\Delta}_{1}=\vec{j} l_{1} \varphi \cos \left(\frac{\varphi}{2}+\beta_{1}\right)+\vec{k}\left[z+l_{1} \varphi \sin \left(\frac{\varphi}{2}+\beta_{1}\right)\right], \\
& \vec{\Delta}_{2}=\vec{j} l_{2} \varphi \cos \left(\beta_{2}-\frac{\varphi}{2}\right)+\vec{k}\left[z-l_{2} \varphi \sin \left(\beta_{2}-\frac{\varphi}{2}\right)\right] ;
\end{aligned}
$$

relative rates of points, which coincide with the ends of the mentioned vectors $\left(\vec{\Delta}_{1}, \vec{\Delta}_{2}\right)$ -

$$
\begin{aligned}
& \dot{\vec{\Delta}}_{1}=\vec{j} l_{1} \dot{\varphi} \cos \left(\frac{\varphi}{2}+\beta_{1}\right)-\vec{j} l_{1} \varphi \frac{\dot{\varphi}}{2} \sin \left(\frac{\varphi}{2}+\beta_{1}\right)+ \\
& +\vec{k}\left[\dot{z}+l_{1} \dot{\varphi} \sin \left(\frac{\varphi}{2}+\beta_{1}\right)+l_{1} \dot{\varphi} \frac{\varphi}{2} \cos \left(\frac{\varphi}{2}+\beta_{1}\right)\right], \\
& \dot{\vec{\Delta}}_{2}=\vec{j} l_{2} \dot{\varphi} \cos \left(\beta_{2}-\frac{\varphi}{2}\right)-\vec{j} l_{2} \varphi \frac{\dot{\varphi}}{2} \sin \left(\beta_{2}-\frac{\varphi}{2}\right)+ \\
& +\vec{k}\left[\dot{z}-l_{2} \dot{\varphi} \sin \left(\beta_{2}-\frac{\varphi}{2}\right)+l_{2} \dot{\varphi} \frac{\varphi}{2} \cos \left(\beta_{2}-\frac{\varphi}{2}\right)\right] ;
\end{aligned}
$$

elastic elements $\delta_{1}, \delta_{2}$ deformations:

$$
\begin{aligned}
& \delta_{1}=\sqrt{\left(z^{*}\right)^{2}+l_{1}^{2} \varphi^{2}+2 z * l_{1} \varphi \sin \left(\frac{\varphi}{2}+\beta_{1}\right)}, \\
& \delta_{2}=\sqrt{\left(z^{*}\right)^{2}+l_{2}^{2} \varphi^{2}-2 z * l_{2} \varphi \sin \left(\beta_{2}-\frac{\varphi}{2}\right)},
\end{aligned}
$$

where $z^{*}=z-\Delta_{s t}, \Delta_{s t}$ - static deformation of the elastic elements, $l_{1}, l_{2}, \beta_{1}, \beta_{2}$-geometric parameters, which specify the location of elastic elements relatively the masses center $\vec{k}, \vec{j}$ - unit vectors directed along the axis stable reading system $\mathrm{OZ}$ and $\mathrm{OY}$ correspondingly (Figure 1). In the case of symmetric location of the cushioned area weight centre relatively the elastic elements $l_{1}=l_{2}$ and $\beta_{1}=\beta_{2}$.

Equations (2)-(4), as well as the assumptions as to the power characteristics of the cushioning system (See 3 ), make possible to present the active force components acting on the CM ( $\vec{F}_{1}$ and $\vec{F}_{2}$ ) as follows:

$$
\begin{aligned}
& \vec{F}_{1}=\delta_{1}^{v_{2}}\left(\alpha_{1}+\chi_{1} \dot{\Delta}_{1}^{v_{1}}\right) . \\
& \left(\vec{j} l_{1} \varphi \sin \left(\frac{\varphi}{2}+\beta_{1}\right)+\vec{k}\left(z^{*}+l_{1} \varphi \sin \left(\frac{\varphi}{2}+\beta_{1}\right)\right)\right), \\
& \vec{F}_{2}=\delta_{2}^{v_{2}}\left(\alpha_{2}+\chi_{2} \dot{\Delta}_{2}^{v 1}\right) . \\
& \cdot\left(\vec{j} l_{2} \varphi \sin \left(\beta_{2}-\frac{\varphi}{2}\right)+\vec{k}\left[z^{*}-l_{2} \varphi \sin \left(\beta_{2}-\frac{\varphi}{2}\right)\right]\right) .
\end{aligned}
$$

The projection sum of the mentioned above forces and the cushioned area weight force on the vertical axis (CZ) will be nothing else but the active component of the generalized force, which corresponds to the generalized coordinate $z$ that is,

$$
\begin{aligned}
& Q_{z}^{a}=\delta_{1}^{v_{2}}\left(\alpha_{1}+\chi_{1} \dot{\Delta}_{1}^{v_{1}}\right)\left(z^{*}+l_{1} \varphi \sin \left(\frac{\varphi}{2}+\beta_{1}\right)\right)+ \\
& +\delta_{2}^{v_{2}}\left(\alpha_{2}+\chi_{2} \dot{\Delta}_{2}^{v_{1}}\right)\left(z^{*}-l_{2} \varphi \sin \left(\beta_{2}-\frac{\varphi}{2}\right)\right)-P .
\end{aligned}
$$

The active component of the generalized force, which corresponds to the generalized coordinates $\varphi$ that is, $Q_{\varphi}^{a}$ is found based on the following [14]: - from one side, the work of active forces acting on the $\mathrm{CM}$, at the possible displacement of the mentioned generalized coordinate $\delta \varphi$, is found by the ratio $\delta A_{\varphi}^{a}=Q_{\varphi}^{a} \delta \varphi$, from the other - $\delta A_{\varphi}^{a}=M_{C}^{a} \delta \varphi$, where $M_{C}^{a}$ - the sum of active forces moments acting on the cushioned area relatively the mass centre. The last value, according to the Varignon theorem [14] is found due to the dependence $M_{C}^{a}=F_{1 z}^{a} y_{1}-F_{1 y}^{a} z_{1}+F_{2 z}^{a} y_{2}-F_{2 y}^{a} z_{2}$, where $F_{1 y}^{a}, F_{1 z}^{a}$ and $F_{2 y}^{a}, F_{2 z}^{a}$ - the projections on the axis $\mathrm{OY}$ and $\mathrm{OZ}$ of the active forces acting on the right and left areas of the CA WV; $y_{1}, z_{1}$ and $y_{2}, z_{2}$ - coordinates of these forces application points, that is, $y_{1}=l_{1} \sin \beta_{1}, z_{1}=-l_{1} \cos \beta_{1}$, $y_{2}=l_{2} \sin \beta_{2}, z_{2}=-l_{2} \cos \beta_{2}$.

Thus, $Q_{\varphi}^{a}=F_{1 z}^{a} y_{1}-F_{1 y}^{a} z_{1}+F_{2 z}^{a} y_{2}-F_{2 y}^{a} z_{2}$.

If corresponding values are substituted in the last dependence, one will obtain:

$$
\begin{aligned}
& Q_{\varphi}^{a}=\left(\alpha_{1}+\chi_{1} \dot{\Delta}_{1}^{v 1}\right) . \\
& \left(\left(z^{*}\right)^{2}+l_{1}^{2} \varphi^{2}+2 z * l_{1} \varphi \sin \left(\frac{\varphi}{2}+\beta_{1}\right)\right)^{\frac{v 2}{2}} \\
& \left\{\begin{array}{l}
l_{1} \sin \beta_{1}\left(z^{*}+l_{1} \varphi \sin \left(\frac{\varphi}{2}+\beta_{1}\right)\right)+ \\
+l_{1} \cos \beta_{1}\left(z^{*}+l_{1} \varphi \sin \left(\frac{\varphi}{2}+\beta_{1}\right)\right)
\end{array}\right\}+ \\
& +\left(\alpha_{2}+\chi_{2} \dot{\Delta}_{2}^{v 1}\right)\left(\begin{array}{l}
\left(z^{*}\right)^{2} l_{2}^{2} \varphi^{2}- \\
-2 z^{*} l_{2} \varphi \sin \left(\beta_{2}-\frac{\varphi}{2}\right)
\end{array}\right)^{\frac{v 2}{2}} \\
& \left\{\begin{array}{l}
l_{2} \sin \beta_{2}\left(z^{*}+l_{2} \varphi \sin \left(\beta_{2}-\frac{\varphi}{2}\right)\right)+ \\
+l_{2} \cos \beta_{2}\left(z^{*}+l_{2} \varphi \sin \left(\beta_{2}-\frac{\varphi}{2}\right)\right)
\end{array}\right\} .
\end{aligned}
$$

Following the similar way as for the active components of the recovering force one will obtain the passive components of the generalized forces

$$
\begin{aligned}
& Q_{i z}^{\Pi}=-\gamma_{i}\left|\bar{f}_{i}(\varphi, \dot{z}, \dot{\varphi})\right| \times \\
& \times\left[\begin{array}{c}
2 \dot{z}+\dot{\varphi}\left(l_{1} \sin \left(\frac{\varphi}{2}+\beta_{1}\right)-l_{2} \sin \left(\beta_{2}-\frac{\varphi}{2}\right)\right)+ \\
+\varphi \frac{\dot{\varphi}}{2}\left(l_{1} \cos \left(\frac{\varphi}{2}+\beta_{1}\right)+l_{2} \cos \left(\beta_{2}-\frac{\varphi}{2}\right)\right)
\end{array}\right], \\
& Q_{\varphi}^{\Pi}=-\gamma_{i}\left|\bar{f}_{i}(\varphi, \dot{z}, \dot{\varphi})\right| \times \\
& \times\left\{l_{1}\left[\dot{z}+\dot{\varphi} l_{1}\left(\sin \left(\frac{\varphi}{2}+\beta_{1}\right)+\frac{\varphi}{2} \cos \left(\frac{\varphi}{2}+\beta_{1}\right)\right)\right] \times\right. \\
& \times\left(\sin \beta+\varphi \cos \left(\frac{\varphi}{2}+\beta_{1}\right)\right)- \\
& -l_{1}^{2}\left[\dot{\varphi}\left(\cos \left(\frac{\varphi}{2}+\beta_{1}\right)-\frac{\varphi}{2} \sin \left(\frac{\varphi}{2}+\beta_{1}\right)\right)\right] \times \\
& \times\left(\cos \beta-\varphi \sin \left(\frac{\varphi}{2}+\beta_{1}\right)\right)+ \\
& +l_{2}\left[\dot{z}-\dot{\varphi} l_{2}\left(\sin \left(\beta_{2}-\frac{\varphi}{2}\right)-\frac{\varphi}{2} \cos \left(\beta_{2}-\frac{\varphi}{2}\right)\right)\right] \times \\
& \times\left(\sin \beta_{2}-\varphi \cos \left(\beta_{2}-\frac{\varphi}{2}\right)\right)- \\
& -l_{2}^{2}\left[\dot{\varphi}\left(\cos \left(\beta_{2}-\frac{\varphi}{2}\right)+\frac{\varphi}{2} \sin \left(\beta_{2}-\frac{\varphi}{2}\right)\right)\right] \times \\
& \left.\times\left(\cos \beta_{2}-\varphi \sin \left(\beta_{2}-\frac{\varphi}{2}\right)\right)\right\} .
\end{aligned}
$$

In the presented above ratio $\bar{f}_{i}(\varphi, \dot{z}, \dot{\varphi})$ - the known functions specified according to the assumptions dealing with the damping device resistance forces. Thus, the vibration process of the cushioned mass is described by the system of differential equations in which the right-hand parts are found according to the Equations (6)-(8). 
$M \frac{d^{2} z}{d t^{2}}=Q_{z}^{a}+Q_{z}^{\Pi}$

$I_{C} \frac{d^{2} \varphi}{d t^{2}}=Q_{\varphi}^{a}+Q_{\varphi}^{\Pi}$

To obtain the analytical solution of the obtained system of non-linear differential equations is not possible. That is why let us analyze only the CM vertical and rolling vibrations separately.

\section{The cushioning mass (CM) vertical vibrations}

These vibrations occur when their perturbation is caused by the bump road, similar under the right and the left wheels. In the mathematical model of the CM dynamics it equals $\varphi \equiv 0$. The mentioned factor, as well as the conditions combined with the power factors acting on the WV CM, make possible to present the first part of the differential system in Equation (9) as follows:

$$
\begin{aligned}
& \ddot{z}^{*}+\frac{\chi_{1}+\chi_{2}}{M}\left(z^{*}\right)^{v_{2}+1}\left(\dot{z}^{*}\right)^{v_{1}}= \\
& =-g+\frac{\alpha_{1}+\alpha_{2}}{M}\left(z^{*}\right)^{v_{2}+1}-\frac{\gamma_{1}+\gamma_{2}}{M}\left|\dot{z}^{*}\right|^{s} \dot{z}^{*} .
\end{aligned}
$$

It should be noted that the maximum value of the right-hand part of the differential equation is a small value in comparison to the maximum value of the function $g(z, \dot{z})=\frac{\chi_{1}+\chi_{2}}{M}\left(z^{*}\right)^{v_{2}+1}\left(\dot{z}^{*}\right)^{v_{1}}$. All the mentioned above makes possible to apply the general rules of the non-linear differential equations perturbation theory with "small" right-hand part [15]. Effectiveness of application of the mentioned methods depends on the possibility to build a real solution of the non-perturbated option of the equation in question. For the non-linear differential $\mathrm{CM}$ vertical vibrations this equation looks like

$\ddot{z}^{*}+\frac{\chi_{1}+\chi_{2}}{M}\left(z^{*}\right)^{v_{2}+1}\left(\dot{z}^{*}\right)^{v_{1}}=0$.

Both the perturbated and non-perturbated differential equations have to describe the $\mathrm{CM}$ vibration processes and the necessary condition for this type of solution in Equation (11) is non-conjugated with respect to the generalized coordinate non-conservative recovering force that is, $\left(-z^{*}\right)^{v_{2}+1}\left(\dot{z}^{*}\right)^{v_{1}}=-\left(z^{*}\right)^{v_{2}+1}\left(\dot{z}^{*}\right)^{v_{1}}$. Thus, the differential Equations (11) and (10) will describe the vibration process, if $v_{i}+1=\left(2 p_{i}+1\right) /\left(2 q_{i}+1\right), p_{i}, q_{i}=0,1,2, \ldots$. Having provided the above mentioned condition, the periodic solution of Equation (11) is described by Equation (13) taking advantage of the periodic Ateb-function [16] as follows

$\left.z^{*}(t)=a s a\left(v_{2}+1, \frac{1}{1-v_{1}}, \omega(a) t+\theta\right)\right)$,

where $a$ - amplitude, $\omega(a) t+\theta$ - phase, $\theta$ - initial phase, $\omega(a)$ - natural frequency of vibration. The latter, being dependent on amplitude for the most of non-linear vibration systems, is found by relation $\omega(a)=\frac{v_{2}+2}{2}\left(\frac{2-v_{1}}{\left(1-v_{1}\right)\left(v_{2}+2\right)} \frac{\chi_{1}+\chi_{2}}{M}\right)^{\frac{1}{2-v_{1}}} a^{\frac{v_{1}+v_{2}}{2-v_{1}}}$.

If in the expression for the natural frequencies of the vertical vibrations more convenient notion "rigidity" is used, that is the static deformation, one will obtain

$\omega(a)=\frac{v_{2}+2}{2}\left(\begin{array}{l}\left.\frac{2-v_{1}}{\left(1-v_{1}\right)\left(v_{2}+2\right)} \times\right)^{\frac{1}{2-v_{1}}} \\ \times \frac{\left(\chi_{1}+\chi_{2}\right) g}{\left(\alpha_{1}+\alpha_{2}\right) \Delta_{\tilde{n} o^{\prime}}^{v_{2}+1}}\end{array}\right)^{\frac{v_{1}+v_{2}}{2-v_{1}}}$.

Reliability of the presented above results can be described as follows: at $v_{1}=0$ the known values can be found, which deal with the non-linear conservative [12], and at $v_{1}=v_{2}=0$ - linear characteristics of the elastic absorbers. Besides that, at $v_{1}=v_{2}$ the CM natural frequency does not depend on the amplitude. It means that in the case of the non-linear non-conservative cushioning system (CS) force, which is changed according to the law $F_{i}\left(\Delta_{i}, \frac{d \Delta_{i}}{d t}\right)=\left(\alpha_{i}+\chi_{i}\left(\frac{d \Delta_{i}}{d t}\right)^{v}\right) \Delta_{i}^{1-v}$, the CS dynamic process is isosynchronic.

The dependencies obtained above are the basis for creation of the software product for control of the nonlinear non-conservative recovering force of the absorbers to provide the most comfortable conditions of people transporting. It is known that the most comfortable conditions of transporting are those, when the vibration frequency of the WV CM varies within $0.8<f<1.5 \mathrm{~Hz}$, [17-18]. That is why, if the main factor of the frequency control is the static deformation of the cushioning system, it is found as the function of the vertical vibrations amplitude according to the relation

$\Delta_{s t .}=\left[\begin{array}{l}\frac{\left(2-v_{1}\right)\left(\chi_{1}+\chi_{2}\right) g}{\left(1-v_{1}\right)\left(v_{2}+2\right)\left(\alpha_{1}+\alpha_{2}\right)} \times \\ \times\left(\frac{\left(v_{2}+2\right)}{4 \Pi f}\right)^{2-v_{1}} a^{v_{1}+v_{2}}\end{array}\right]^{\frac{1}{v_{2}+1}}$,

where $\Pi=\frac{\Gamma\left(1 /\left(v_{2}+2\right)\right) \Gamma\left(\left(1-v_{1}\right) /\left(2-v_{1}\right)\right)}{\Gamma\left(1 /\left(v_{2}+2\right)+\left(1-v_{1}\right) /\left(2-v_{1}\right)\right)}$ - the semi-period according to the phase of the used special Ateb-functions.

In Figure 2 it is shown, according to Equation (14), the laws of the CM static deformation change (the main parameter of the guided CS, at which the frequency of the natural CM vibrations is of the most comfortable for transportation of dangerous goods and passengers.

Presented graphic dependencies show that in order to meet the ergonomic requirements as to the frequency of the vertical vibrations, in the case of the great $\mathrm{CM}$ vibration amplitudes $(a>0.1 \mathrm{~m})$ it is recommended to choose the CS with the static deformation $0.2<\Delta_{\text {st }}<0.35$ in the cases of the progressive CS power characteristics 


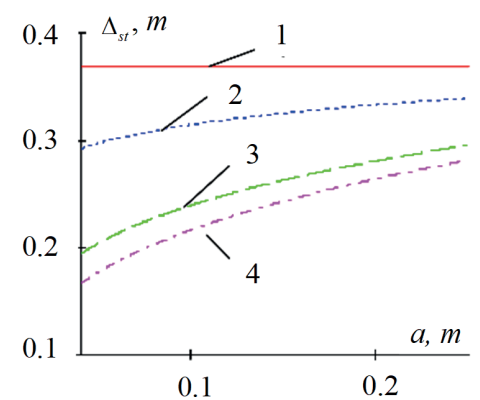

$v_{1}=-2 / 7 ; 1-v_{1}=2 / 7 ; 2-v_{2}=2 / 5$

$3-v_{3}=2 / 3 ; 4-v_{4}=4 / 5$

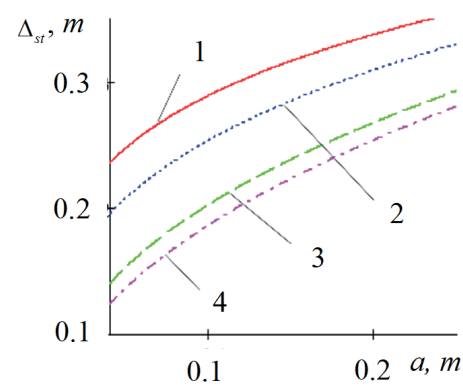

$v_{1}=0 ; 1-v_{1}=2 / 7 ; 2-v_{2}=2 / 5$

$3-v_{3}=2 / 3 ; 4-v_{4}=4 / 5$

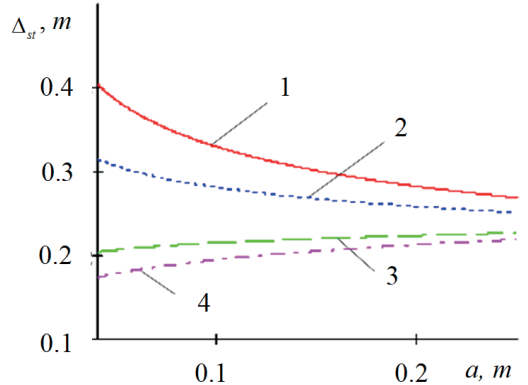

$v_{1}=-4 / 7 ; 1-v_{1}=2 / 7 ; 2-v_{2}=2 / 5$

$3-v_{3}=2 / 3 ; 4-v_{4}=4 / 5$

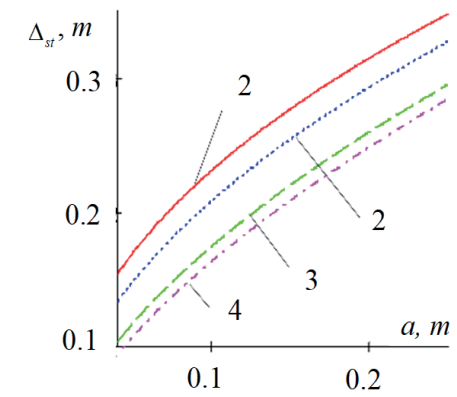

$v_{1}=2 / 7 ; 1-v_{1}=2 / 7 ; 2-v_{2}=2 / 5$

$3-v_{3}=2 / 3 ; 4-v_{4}=4 / 5$

Figure 2 Dependence of the static deformation on amplitude of the vertical vibrations of the WV guided CS with the non-conservative power characteristics of the cushioning system

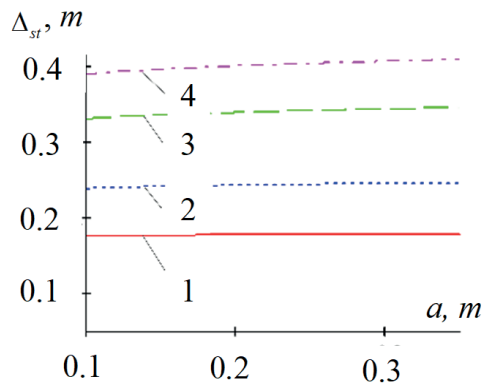

$v_{2}=0 ; f=1.5 ; 1-v_{1}=2 / 13$;

$2-v_{2}=4 / 13 ; 3-v_{3}=6 / 13$;

$4-v_{4}=8 / 15$

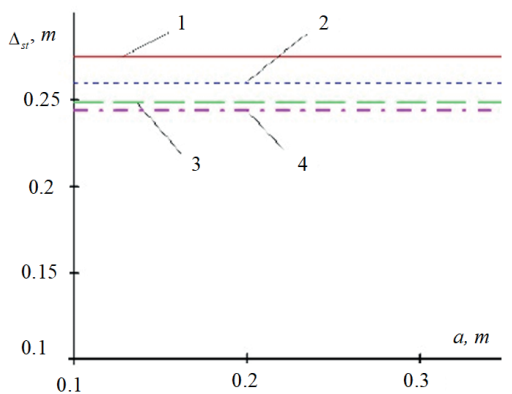

$v_{2}>0 ; f=1.0 ; 1-v_{1}=2 / 13$;

$2-v_{2}=4 / 13 ; 3-v_{3}=6 / 13$;

$$
4-v_{4}=8 / 15
$$

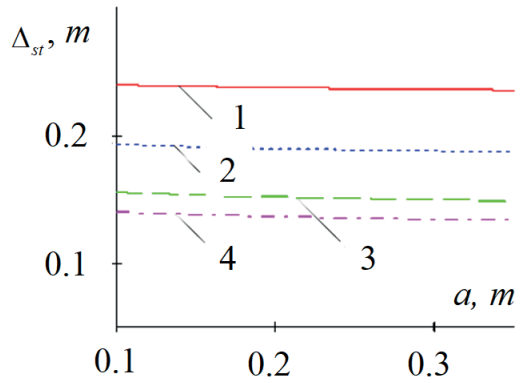

$v_{2}=0 ; f=1.0 ; 1-v_{1}=2 / 13$;

$2-v_{2}=4 / 13 ; 3-v_{3}=6 / 13$;

$4-v_{4}=8 / 15$

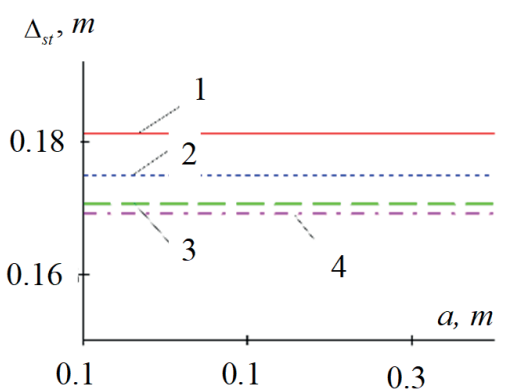

$v_{2}>0 ; f=1.25 ; 1-v_{1}=2 / 13$;

$2-v_{2}=4 / 13 ; 3-v_{3}=6 / 13 ;$

$4-v_{4}=8 / 15$

Figure 3 Dependence of the static deformation on the vertical vibration amplitude of the WV guided $C S v_{1}=-v_{2}$ and $v_{2}=0,0<v_{1}<1$ 

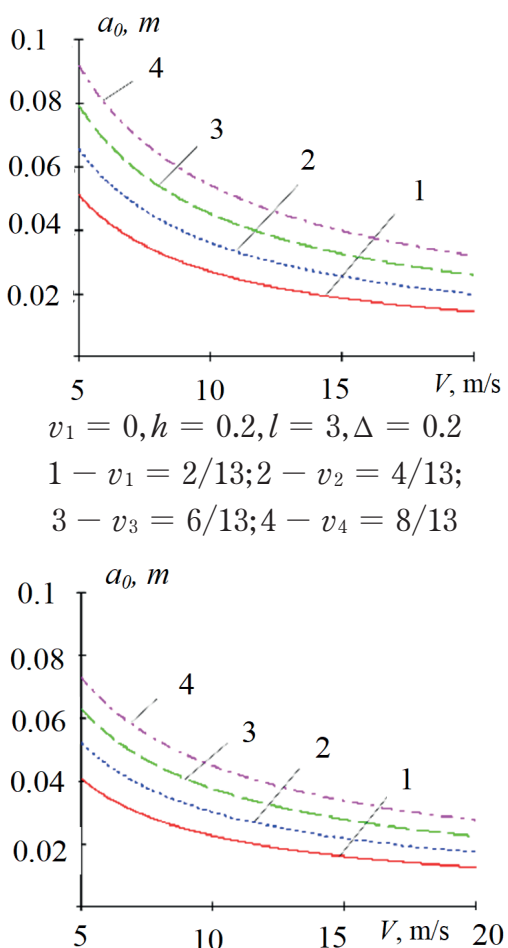

$v_{1}=2 / 13, h=0.2, l=3, \Delta=0.15$

$1-v_{1}=2 / 13 ; 2-v_{2}=4 / 13$;

$3-v_{3}=6 / 13 ; 4-v_{4}=8 / 13$

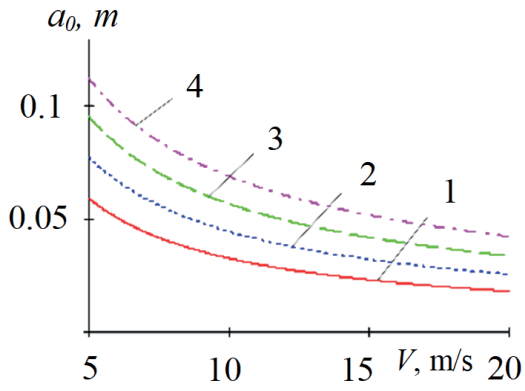

$v_{1}=2, h=0.2, l=3, \Delta=0.3$

$1-v_{1}=2 / 13 ; 2-v_{2}=4 / 13$;

$3-v_{3}=6 / 13 ; 4-v_{4}=8 / 13$

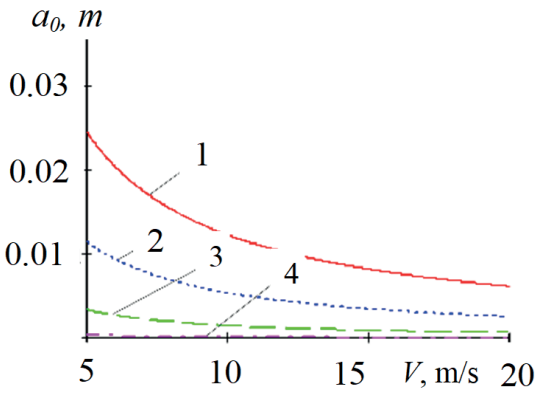

$v_{1}=2 / 13, h=0.2, l=3, \Delta=0.3$

$1-v_{1}=2 / 13 ; 2-v_{2}=4 / 13$

$3-v_{3}=6 / 13 ; 4-v_{4}=8 / 13$

Figure 4 Dependences of the initial perturbation amplitude on the speed of getting into the bump

(at $v_{1}=0 ;-0<v_{2}<1$ ) and non-conservative at $-0.4<v_{1}<0 ; 0<v_{2}<1$. What concerns the small vibration amplitudes, it is necessary for the progressive cushioning system and non-conservative one (with the parameter values $-0.4<v_{1}<1,0<v_{2}<1$ ) that the cushioning system static deformation of elastic absorbers was varying within $0.1<\Delta_{\text {st }}<0.2$ and in the case $\quad-0.7<v_{1}<-0,4,0<v_{2}<1 \quad$ within $0.25<\Delta_{\text {st }}<0.4 \mathrm{~m}$. The CS with values of the power parameters of its non-conservative recovering force $v_{1}=-v_{2}$ and $v_{2}=0,0<v_{1}<1$ is of the great practical importance, which is seen in Figure 3. The ergonomic requirements for such a $\mathrm{CS}$ as to the vibration frequencies are provided, when the static deformation is stable and does not depend on the $\mathrm{CM}$ vibration amplitudes (Figure 3 ).

The dependencies obtained above can be the basis for finding the amplitude of the initial perturbation of the vertical vibrations under the condition when the bump road under the right and left wheels are of similar profiles. If one assumes that the latter are described by relation $z=h \sin \frac{\pi}{l} x$ and that the WV moves with the constant speed $\mathrm{V}$, then the relative component of the initial $\mathrm{CM}$ speed from the one side is equal $V_{0}=V \sin \alpha_{0},\left(\alpha_{0}\right.$ the tangent inclination angle to the bump at the moment, when the wheel gets into the bump) and from the other $V_{0}=\frac{d z}{d t}_{t=0}^{*}=\frac{2 a_{0}}{v_{2}+2} \omega\left(a_{0}\right)$. All the presented makes possible the obtain Equation (15) for finding the amplitude of the initial perturbation $a_{0}$ in the case of the slope road bump:

$$
\begin{aligned}
& a_{0}=\left(V \frac{h \pi}{l}\right)^{\frac{2-v_{1}}{2+v_{2}}} \times \\
& \times\left(\frac{\left(1-v_{1}\right)\left(v_{2}+2\right)\left(\alpha_{1}+\alpha_{2}\right) \Delta_{c m .}^{v_{2}+1}}{)^{\frac{1}{v_{2}+2}} .} .\right.
\end{aligned}
$$

According to dependencies in Equation (15), dependence of the amplitude of the initial perturbation $\left(a_{0}=a_{1}(x)\right)$ on the motion speed $\mathrm{V}$ is presented in Figure 4 and on the static deformation in Figure 5, at different values of the WV CS power.

The presented dependencies show that the amplitude of the WV CM initial vibration perturbation during getting into the bump:

- $\quad$ is smaller for the greater motion speed;

- greater values of the parameter $v_{2}$, correspond to the greater values of the initial perturbation amplitude in the case of the progressive or regressive power characteristics;

- for the case $v_{1}>0$ and $v_{2}>0 ; v_{1}<0$ and $v_{2}>0$, the amplitude of the initial perturbation is greater than the amplitude of the CS initial perturbation with the CS linear characteristics (at all other parameters being constant), and for the cases $v_{1}<0$ and $v_{2}<0$; $v_{1}>0$ and $v_{2}<0$ it is smaller;

- the greater values of the elastic absorbers static deformation of the cushioning system correspond to the greater values of the initial perturbation amplitudes. Moreover, for its progressive power characteristics and for the cases when $v_{1}>0$ and $v_{2}>0 ; v_{1}<0$ and $v_{2}>0$, it is greater than the amplitude of the CS initial 

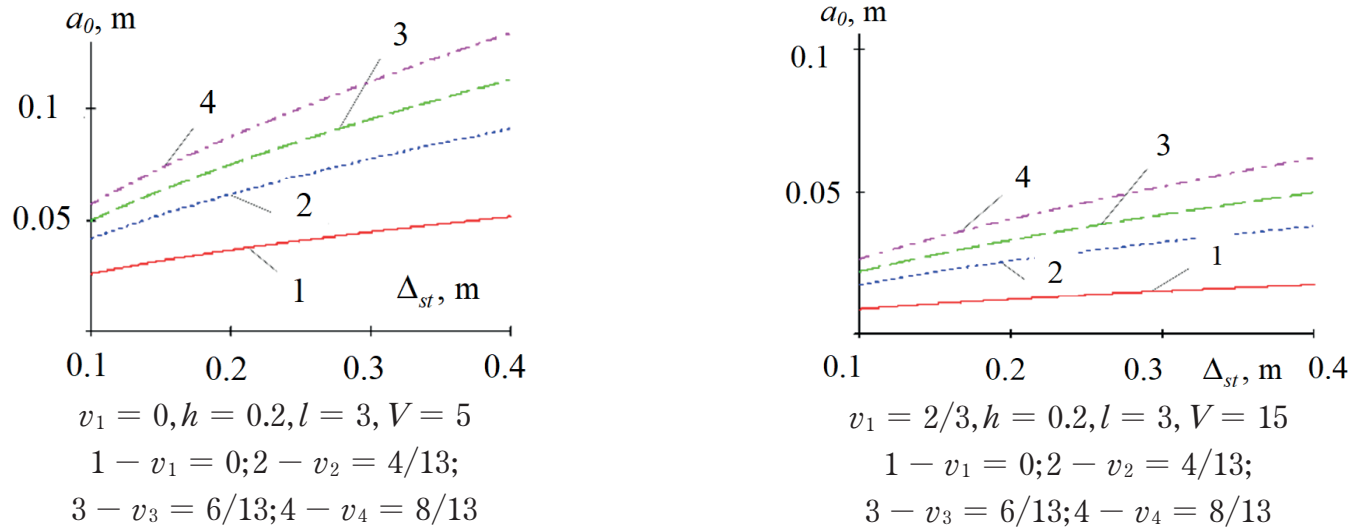

Figure 5 Dependences of the initial perturbation amplitude on the CS static deformation
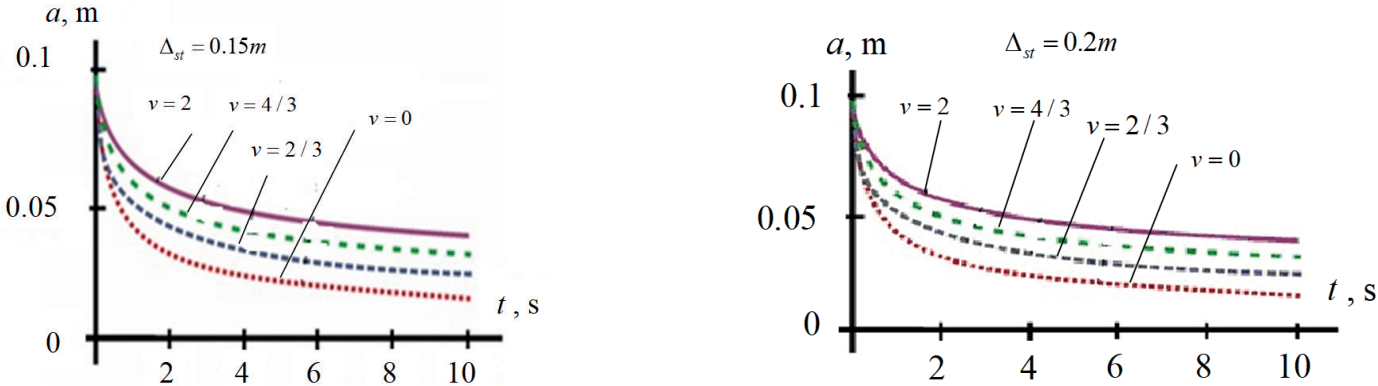

Figure 6 Time changes of the damped vertical vibrations amplitude at different values of the power characteristics of the guided CS

perturbation with the linear power characteristics and for the regressive one and the cases $v_{1}<0$ and $v_{2}<0 ; v_{1}>0$ and $v_{2}<0-$ it is smaller.

As to the effect of the "small" non-linear resistance forces of the WV CM dynamics, it is demonstrated in the change in time of both the amplitude and the vibration frequencies. To state the law of the mentioned parameters change, advantage of the Vander Paul method was employed to Equation (10) and the following was obtained [19-22]:

$$
\begin{aligned}
& \frac{d a}{d t}=\frac{2-v_{1}}{4 \Pi \omega(a)} \int_{0}^{2 \Pi}\left(c a\left(\frac{1}{1-v_{1}}, v_{2}+1, \psi\right)\right) \times \\
& \times\left\{\begin{array}{l}
-g+\frac{\alpha_{1}+\alpha_{2}}{M} a^{v 2+1} s a^{v^{2}+1} \times \\
\times\left(v_{2}+1, \frac{1}{1-v_{1}}, \psi\right)+\frac{\gamma_{1}+\gamma_{2}}{M} \times \\
\times \mid \frac{2 a(t) \omega(a)}{v_{2}+2}\left(c a\left(\frac{1}{1-v_{1}}, v_{2}+1, \psi\right)\right)^{\frac{1}{1-v 1}} \\
\frac{2 a(t) \omega(a)}{v_{2}+2}\left(c a\left(\frac{1}{1-v_{1}}, v_{2}+1, \psi\right)\right)^{\frac{1}{1-v 1}}
\end{array}\right\} d \psi,
\end{aligned}
$$$$
\frac{d \psi}{d t}=\omega(a)-\frac{\alpha_{1}+\alpha_{2}}{M} \frac{\left(v_{2}+2\right)\left(2-v_{1}\right) a^{v 2}}{4 \Pi \omega(a)} \times
$$$$
\times \frac{2 \Gamma\left(\frac{2 v_{1}-1}{v_{1}-2}\right) \Gamma\left(\frac{v_{2}+3}{v_{2}+2}\right)}{\Gamma\left(\frac{2 v_{1}-1}{v_{1}-2}+\frac{v_{2}+3}{v_{2}+2}\right)} .
$$

In Figure 6 the laws of the CM vertical vibrations amplitude change in time, for some values of the power characteristics of the guided cushioning system, are presented.

The graph dependencies show that the qualitative nature of the $\mathrm{CM}$ vibration damping remains, but the amplitude damping rate greatly depends on the power characteristics of the absorbers.

\section{The CM rolling vibrations}

These vibrations occur under the condition when the CM motion perturbation was caused by getting on single bump by the right (left) WV tire. The mathematical model of the mentioned WV area vibration can be easily obtained from Equations (7) - (9), when $z^{*}(t) \equiv \Delta_{s t}$. Only the nonperturbated equation, which corresponds to the WC $\mathrm{CM}$ rolling vibration, is presented below

$$
I_{0} \ddot{\varphi}+\Xi^{2} \dot{\varphi}^{v 1} \varphi^{v 2}=0,
$$

where $\Xi^{2}=\left(\chi_{1} l_{1}^{v 1+v 2+2} \sin \beta_{1}\left(\sin \beta_{1}+\cos \beta_{1}\right)+\right.$ $\left.+\chi_{2} l_{2}^{v 1+v 2+2} \sin \beta_{2}\left(\sin \beta_{2}+\cos \beta_{2}\right)\right)$.

The differential equation (18) is similar to Equation (10), according to its structure, that is why the method of its solution is not mentioned here, but the main relations, which describe the main characteristics of the rolling vibration, are presented. Thus, the natural frequency of these vibrations $\Omega$, as a function of the rolling vibrations amplitude $a_{\varphi}$ is found by relation 


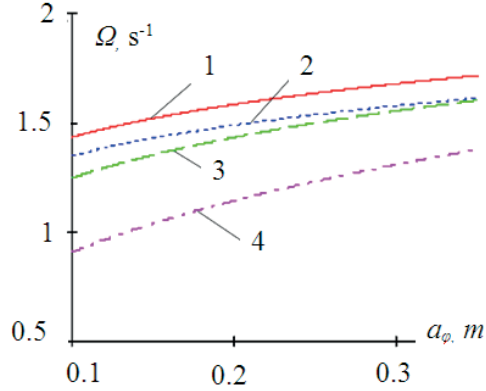

$\Delta_{s t}=0.15 ; \rho=1.4 ; a=b=1.1, v_{1}=0$;

$1-v_{1}=2 / 9 ; 2-v_{2}=2 / 7 ; 3-v_{3}=2 / 5$

$$
4-v_{4}=2 / 3
$$

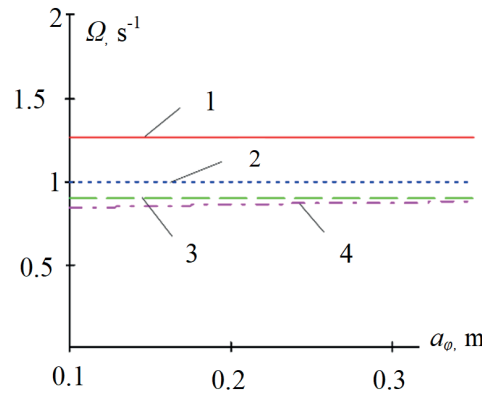

$\Delta_{s t}=0.25 ; \rho=1.4 ; a=b=1.1, v_{1}>0$;

$1-v_{1}=2 / 9 ; 2-v_{2}=2 / 7 ; 3-v_{3}=2 / 5$;

$4-v_{4}=2 / 3$

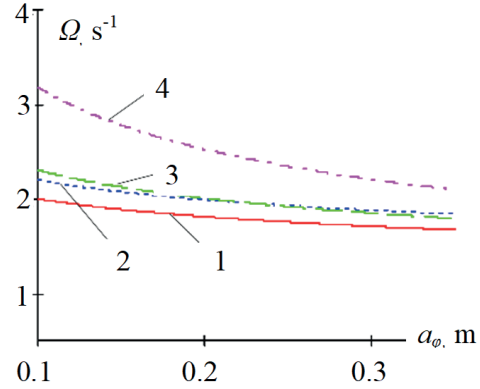

$\Delta_{s t}=0.30 ; \rho=1.4 ; a=b=1.1, v_{1}=0$;

$1-v_{1}=2 / 9 ; 2-v_{2}=2 / 7 ; 3-v_{3}=2 / 5$

$4-v_{4}=2 / 3$

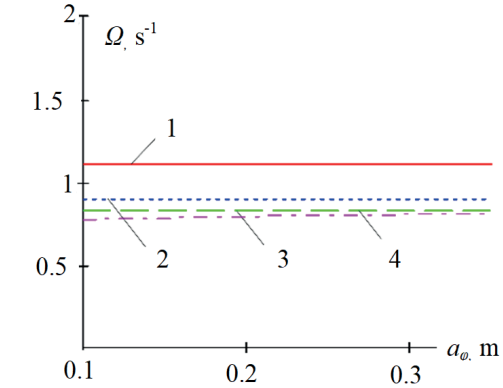

$\Delta_{s t}=0.35 ; \rho=1.4 ; a=b=1.1, v_{1}>0$;

$1-v_{1}=2 / 9 ; 2-v_{2}=2 / 7 ; 3-v_{3}=2 / 5$

$4-v_{4}=2 / 3$

Figure 7 Dependence of the rolling vibration frequency on amplitude at different values of the power characteristics of the guided cushioning system

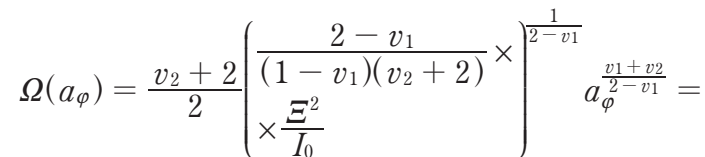

$=\frac{v_{2}+2}{2}\left(\begin{array}{l}\frac{2-v_{1}}{\left(1-v_{1}\right)\left(v_{2}+2\right)} \times \\ \times \frac{\Xi^{2} g}{\left(\alpha_{1}+\alpha_{2}\right) \Delta_{s t .}^{v 2+1} \rho^{2}}\end{array}\right)^{\frac{1}{2-v_{1}}} a_{\varphi}^{\frac{v 1+v 2}{2-v_{1}}}$,

where $\rho$ - CM inertia radius with respect to the longitudinal axis, which crosses the masses centre. In Figure 7 the dependence of the natural frequency of the rolling vibrations in $\mathrm{H} 3 \Omega\left(a_{\varphi}\right) / 2 \Pi$ on the amplitude of the mentioned vibrations, at different values of the CS power characteristics and its static deformation, are presented.

From the presented graphic dependencies can be seen that:

- for the progressive CS power characteristics, the greater vibration amplitude (vertical or rolling) is, the lesser is the natural vibration, moreover, when the values of the CS static deformation are greater (all other values being constant), it is smaller;

- for the regressive CS, the greater are the values of the vibration amplitude, the lesser are the values of the natural vibration frequencies;

- the ergonomic conditions of the WV operation with the progressive CS characteristics in the wide range of the vibration amplitudes are satisfied by the suspension with the static deformation $0.12<\Delta_{\text {st. }}<0.15 \mathrm{~m}$ at $2 / 9<v_{2}<2 / 3$ and for the regressive at $0.2<\Delta_{s t}<0.35 \mathrm{~m},-2 / 3<v_{2}<-2 / 9$ and great values of the rolling vibration amplitudes;

- natural frequency of the CM vibrations with the nonconservative $\mathrm{CS}$ power characteristic at $v_{1}>0$, $v_{2}>0$ and greater vibration amplitudes becomes greater and satisfies the ergonomic conditions at $0.22<\Delta_{s t}<0.35 \mathrm{~m}$;

- for the characteristics at $v_{1}=-v_{2}, v_{2}>0$ in the case $\left|\left(V_{1}\right)\right|>V_{2}$ the greater values of the amplitude correspond to the smaller values of frequency;

- non-conservative CS power characteristics at $V_{1}=-V_{2},-V_{2}>0$ satisfies the ergonomic conditions at $0.15<\Delta_{\text {const }}<0.35 \mathrm{~m}$ at the arbitrary amplitudes of the rolling vibrations.

As to the damping amplitude of the rolling vibrations, their qualitative characteristic is similar to those vertical. That is why they were not analysed.

\section{The effect of the power characteristics of the guided suspension on stability of the WV motion along the curvilinear area of the road}

The WV CM vibrations affect not only the comfortable transporting of people, but the stability of motion while going around the obstacles, manoeuvring motion along the curvilinear areas of the bump road. All mentioned above 

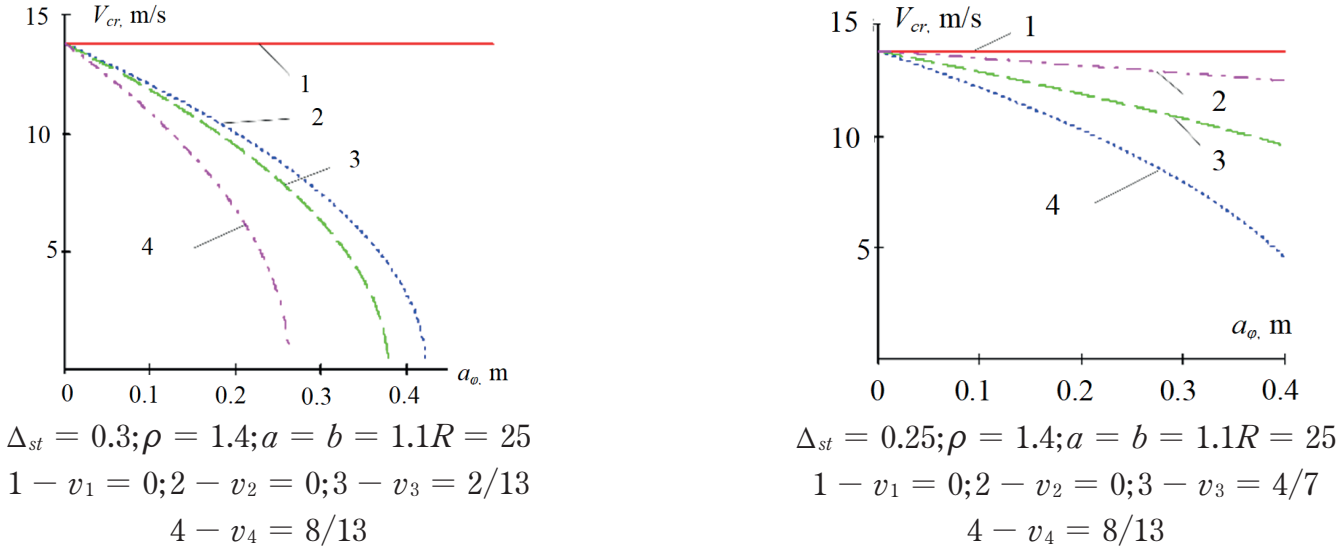

Figure 8 Laws of the critical change of the stable motion speed for capsizing at different values of the CS power characteristics.

is the curvilinear trajectory of motion of the cushionless area weight centre, as well as the point, which coincides with the weight centre of the cushioned area in its transient motion. From the mentioned above it is clear that all the cases of the WV motion are according to the kineticstatistics equations, which besides the active and passive forces take into account the inertia forces of the mentioned areas [14-16, 19]. The main vector of the inertia forces of the cushionless area $\Phi_{Q}^{e}$ (in the case of the WV motion with the constant speed) equals $\Phi_{Q}^{e}=Q V^{2} / g R$ and is directed to the opposite side to the trajectory inner normal of the mentioned point. As to the main vector of the CM inertia forces, is consists of two components: transferred $\Phi_{\Pi}^{e}=Q V^{2} / g R$ and relative $\Phi_{\Pi}^{r}=-\frac{P}{g} \frac{d^{2} z}{d t^{2}}$.

If the first component of the $\mathrm{CM}$ inertia force was found similarly to that cushionless one, as to the direction, the second was by the vertical direction.

Furthermore, the main moment of the inertia force of the $\mathrm{CM}$ relative motion with respect to the arbitrary centre equals $M^{r}=-I_{0} \frac{d^{2} \varphi}{d t^{2}}$, where $I_{0}$ - inertia moment with respect to the mentioned centre.

From the other side, the most dangerous, because of the WV capsizing during the movement along the curvilinear areas of the road, are the rolling vibrations [23-29]. That is why to find the critical speed of the stable motion of the WV with non-conservative power CS characteristics, along the curvilinear area of the road, only the rolling $\mathrm{CM}$ vibrations will be taken into account. In this case, from the kineticstatic equations for the system cushionless-cushioned mass for finding the critical speed of the stable motion against capsizing $V_{\mathrm{cr}}$, one obtains:

$$
\begin{aligned}
& P b+Q \frac{a+b}{2}-I_{A 2} \frac{d^{2} \varphi}{d t^{2}}- \\
& -\frac{P}{g} \frac{\tilde{V}_{c r}^{2}}{R} h_{c}-\frac{Q}{g} \frac{\tilde{V}_{c r}^{2}}{R} r=0,
\end{aligned}
$$

where $I_{A_{2}}$ - inertia moment of the cushioned mass with respect to the contact point of the "outer" tire and the way, which, according to the Huygens-Steiner theorem, equals $I_{A_{2}}=\frac{P}{g}\left(\rho^{2}+\left(\frac{a+b}{2}\right)^{2}+h_{c}^{2}\right), h$-height of the
CM weight centre over the road in the static position.

It is considered that the road curvature radius is much greater than the WV base, that is why it is assumed that the curvature radius of the $\mathrm{WV}$ points in the transient motion is constant.

If in expression for the moment of the inertia forces relatively the $\mathrm{CM}$ motion one uses only the maximum values of the used Ateb-functions, Equation (20) would look like:

$$
\begin{aligned}
& P_{b}+Q \frac{a+b}{2}-I_{A_{2}} \frac{4 a_{\varphi}\left(1-v_{1}\right)}{\left(v_{2}+2\right)\left(2-v_{1}\right)} \Omega^{2} \times \\
& \times\left(a_{\varphi}\right)-\frac{P}{g} \frac{\tilde{V}_{c r}^{2}}{R} h c-\frac{Q}{g} \frac{\tilde{V}_{c r}^{2}}{R} r=0 .
\end{aligned}
$$

The last dependence specifies the critical value of the stable motion speed taking into account the rolling vibrations as follows

$\tilde{V}_{c r}=\sqrt{\frac{-I_{A_{2}} \frac{4 a_{\varphi}\left(1-v_{1}\right)}{\left(v_{2}+2\right) \times\left(2-v_{1}\right)} \Omega^{2}\left(a_{\varphi}\right)}{P h_{c}+Q r}} R g$.

In Figure 8, for some WV geometric and power characteristics, the dependence of the critical speed of the stable motion against the capsizing on the amplitude of the rolling vibrations is presented.

As it was expected, the $\mathrm{CM}$ rolling vibrations cause the decrease of the stable motion critical speed. Besides that, at critical speed of the stable motion due to the capsizing:

- for the progressive or regressive CS power characteristics (at similar amplitudes of the rolling vibrations), the greater values $v_{2}$ correspond to the smaller values of the critical speed;

- for the non-concentrative characteristics at $v_{1}>0$, $v_{2}>0$, the greater values $v_{2}$ correspond to the smaller values of the critical speed;

- in the case of the greater values of the CS static deformation (all other kinematic and power characteristics being constant) the critical value of the stable motion speed is greater. 


\section{Conclusions}

The presented method for investigation of the WV dynamics with the non-linear CS power characteristics made possible to obtain the analytical dependencies, which can be the basis for creation of the software product of the guided WV in order to: meet the ergonomic requirements related to the people transportation; minimize the dynamic loadings on the transported loads; improve the motion stability along the curvilinear areas of the bump road. As to the quantitative recommendations, resulted in the obtained data in order to meet the ergonomic requirements related to the frequency of the vertical vibrations, it is proposed, in the case of the WV movement along the sufficiently bump road, to choose the CS with the static deformation $0.2<\Delta_{s t}<0.35 \mathrm{~m}$. In the case of the progressive power characteristics of the cushioning system at $v_{1}=0 ; 0.1<v_{2}<1$ and non-concentrative at $-0.4<v_{1}<0 ; 0<v_{2}<1$. As to the motion along the road with insufficient bumps - the progressive CS and non-concentrative with values $-0,4<v_{1}<1$ are recommended to be chosen, $0<v_{2}<1$ at the static deformation - $-0.1<\Delta_{s t}<0.2 \mathrm{~m}$. The developed model allows to obtain the parameters of the resonant mode of oscillations, which have their own limiting velocity of the inequality path. The suspension has been designed to minimize the effects of overload caused by the movement of the vehicle on a pavement with irregularities with a frequency that meets the ergonomic conditions for the greatest comfort of transporting people. The following studies for this class of suspensions have been conducted by the authors in subsequent publications.

\section{References}

[1] SOLTUS, A. P. Theory of car performance characteristics. Kyiv, Ukraine: Aristei, 2010. ISBN 966-8458-38-9

[2] SOLIMAN, A., MOUSTAFA, S., SHOGAE, A. Parameters affecting vehicle ride comfort using half vehicle model. SAE Technical Paper [online]. 2008, 2008-01-1146. ISSN 0148-7191, eISSN 2688-3627. Available from: https://doi. org/10.4271/2008-01-1146

[3] DEB, A., JOSHI, D. A study on ride comfort assessment of multiple occupants using lumped parameter analysis. SAE Technical Paper [online], 2012, 2012-01-0053. ISSN 0148-7191, eISSN 2688-3627. Available from: https://doi. org/10.4271/2012-01-0053

[4] ZANDIEH, A. Dynamics of a three - wheel vehicle with tadpole design [online]. A thesis presented to the University of Waterloo in fulfilment of the thesis requirement for the degree of Master of Science in Mechanical Engineering. Ontario, Canada: 2014. Available from: https://uwspace.uwaterloo.ca/bitstream/

[5] PREVIATI, G., GOBBI, M., MASTINU, G. Friction coefficient on snowy and icy surfaces of pneumatic tires fitted with or without anti-skid devices. SAE Technical Paper [online]. 2006, 2006-01-0560. ISSN 0148-7191, eISSN $2688-3627$. Available from: https://doi.org/10.4271/2006-01-0560

[6] GRUBEL, M. H., KRASYUK, O. P., NANIVSKYY, R. A., SOKIL, M. B. Vertical oscillations of wheeled vehicles cushion part under random disturbance conditions. Scientific Notes of Lutsk National Technical University. 2014, 46, p. 112-116. ISSN 2415-3966.

[7] KUZIO, I. V., SOKIL, B. I., PALYUKH, V. M. Suspension parameters effect on wheeled vehicles non-linear oscillations. Bulletin of National University Lviv of Polytechnic, Dynamics, Durability and Design of Machines and Instruments. 2007, 588, p. 49-52. ISSN 0321-0499.

[8] SOKIL, B., LYASHUK, O., PERENCHUK, O., SOKIL, M., POPOVICH, P., VOVK, Y. Dynamic effect of cushion part of wheeled vehicles on their steerability. International Journal of Automotive and Mechanical Engineering [online]. 2018, 15(1), p. 4880-4892. ISSN 2229-8649, eISSN 2180-1606. Available from: https://doi.org/10.15282/ijame.15.1.2018.1.0380

[9] LYASHUK, O., SOKIL, M., VOVK, Y., TSON, O., DZYURA, V. The impact of the kinematic parameters of bounce and pitch motions of sprung mass on wheeled vehicles handling. Scientific Journal of Silesian University of Technology. Series Transport [online]. 2017, 97, p. 81-91. ISSN 0209-3324, eISSN 2450-1549. Available from: https://doi.org/10.20858/ sjsutst.2017.97.8

[10] TAMBOLI, J. A., JOSHI, S. G. Optimum design of a passive suspension system of a vehicle subjected to actual road random excitations. Journal of Sound and Vibration [online]. 1999, 219(2), p. 193-205. ISSN 0022-460X. Available from: https://doi.org/10.1006/jsvi.1998.1882

[11] BOGDEVICIUS, M., JUNEVICIUS, R.: Dynamic processes of the retrofitted rear suspension of the vehicle. Transport [online]. 2004, 19(6), p. 262-268. ISSN 1648-4142. Available from: https://doi.org/10.3846/16484142.2004.9637985

[12] PECELIUNAS, R., LUKOSEVICIENE, O., PRENTKOVSKIS, O. A mathematical model of the vibrating system equivalent to the vehicle in the mode of emergency braking. Transport [online]. 2003, 18(3), p. 136-142. ISSN 1648-4142. Available from: https://doi.org/10.3846/16483840.2003.10414082

[13] LITVINENKO, I.V., MARUSHCHAK, P.O., LUPENKO, S.A. Processing and modeling of ordered relief on the surface of heat-resistant steels after laser irradiation as a cyclic random process. Automatic Control and Computer Sciences [online]. 2014, 48, p. 1-9. ISSN 0146-4116, eISSN 1558-108X. Available from: https://doi.org/10.3103/S0146411614010040 
[14] TAGHAVIFAR, H., MARDANI, A. Off-road vehicle dynamics: analysis, modelling and optimization [online]. Switzerland: Springer, 2017. ISBN 978-3-319-42519-1, eISBN 978-3-319-42520-7. Available from: https://doi.org/10.1007/9783-319-42520-7

[15] GILLESPIE, T. D. Fundamentals of vehicle dynamics. Warrendale, PA: Society of Automotive Engineers, 1992. ISBN 978-1-56091-199-9.

[16] BALTHAZAR, M., MOOK, D. T., WEBER, H. I., BRASIL, R. M. L. R. F., FENILI, A., BELATO D., FELIX, J. L. P. An overview on non-ideal vibrations. Meccanica [online]. 2003, 38(6), p. 613-621. ISSN 0025-6455, eISSN 1572-9648. Available from: https://doi.org/10.1023/A:1025877308510

[17] BLEKHMAN, I. I. Vibrational mechanics: nonlinear dynamic effects, general approach, applications. Singapore: World Scientific, 2000. ISBN 978-9810238902.

[18] SERTA, E., BOYRAZ, P. Optimization of suspension system and sensitivity analysis for improvement of stability in a midsize heavy vehicle. Engineering Science and Technology, an International Journal [online], 2017, 20(3), p. 997-1012. ISSN 2215-0986. Available from: https://doi.org/10.1016/j.jestch.2017.03.007

[19] RILL, G. Road vehicle dynamics: fundamentals and modeling. Boca Raton FL: CRC Press, 2012. ISBN 9781439838983.

[20] ARTYUSHENKO, A., SUYARKOV, O. Study of influence of suspension characteristics of small size car on ride quality and its modernization. Bulletin NTU KhPI. Series: Transport Mechanical Engineering. 2013, 31(1004), p. 21-27. ISSN 2078-6840.

[21] PODRYHALO, M. A., KORBKO, M. I., KLETS, D. M. Evaluation of automobile dynamic ability. Bulletin NTU KhPI, Automobile and Tractor Manufacture. 2008, 58, p. 134-137. ISSN 2078-6840.

[22] PODRYHALO, M. A., VOLKOV, V. P., BOBOSHKO, A. A., PAVLENKO, V. A., BAITSUR, M. V., NAZAROV, A. I., ALEKSEV, V. O. Wheeled vehicles resistance to skidding whilst breaking and ways of its rising. Kharkiv: KNARU, 2006

[23] GRUBEL, M. H., NANIVSKYY, R. A., SOKIL, M. B. Effect of elastic suspension restoring force on wheeled vehicles stable motion. In: International Scientific and Technical Conference: proceedings. Lviv: ACB, 2014.

[24] COLE, J. Disturbance methods in applied mathematics. Moscow: Mir. 1972.

[25] BOHOLYUBOV, N. N., MYTROPOLSKYY, Y. A. Asymptomatic methods in non-linear oscillations theory. Moscow: Science, 1974.

[26] SENYK, P. M. Inversion of the incomplete beta function. Ukrainian Mathematical Journal [online]. 1969, 21(3), p. 271-278. ISSN 0041-5995, eISSN 1573-9376. Available from: https://doi.org/10.1007/BF01085368

[27] SENYK, P. M., SOKIL, B. I. About U-method use for one class of oscillation system. Report Academy of Sciences USSR. 1977, 1, p. 12-16.

[28] SOKIL, B. I., SENYK, P. M. Parameters determination of non-linear oscillation system on amplitude - frequency characteristic. Mathematical Methods and Physical and Mechanical Fields. 1977, 7, p. 94-99.

[29] PAVLENKO, V. M., KRYVORUCHKO, O. O. Modern state of development of active suspensions of motor cars. Bulletin NTU KhPI, Automobile and Tractor Manufacture [online]. 2014, 1052, p. 54-60. ISSN 2078-6840. 